\title{
Front Matter: Volume 7912
}

, "Front Matter: Volume 7912," Proc. SPIE 7912, Solid State Lasers XX: Technology and Devices, 791201 (1 April 2011); doi: 10.1117/12.890277

SPIE. Event: SPIE LASE, 2011, San Francisco, California, United States 


\title{
PROCEEDINGS OF SPIE
}

\section{Solid State Lasers XX: Technology and Devices}

\author{
W. Andrew Clarkson \\ Norman Hodgson \\ Ramesh Shori \\ Editors
}

23-27 January 2011

San Francisco, California, United States

Sponsored and Published by

SPIE 
The papers included in this volume were part of the technical conference cited on the cover and title page. Papers were selected and subject to review by the editors and conference program committee. Some conference presentations may not be available for publication. The papers published in these proceedings reflect the work and thoughts of the authors and are published herein as submitted. The publisher is not responsible for the validity of the information or for any outcomes resulting from reliance thereon.

Please use the following format to cite material from this book:

Author(s), "Title of Paper," in Solid State Lasers XX: Technology and Devices, edited by W. Andrew Clarkson, Norman Hodgson, Ramesh Shori, Proceedings of SPIE Vol. 7912 (SPIE, Bellingham, WA, 2011) Article CID Number.

ISSN 0277-786X

ISBN 9780819484499

Published by

SPIE

P.O. Box 10, Bellingham, Washington 98227-0010 USA

Telephone +1 3606763290 (Pacific Time) · Fax +1 3606471445

SPIE.org

Copyright (C) 2011, Society of Photo-Optical Instrumentation Engineers

Copying of material in this book for internal or personal use, or for the internal or personal use of specific clients, beyond the fair use provisions granted by the U.S. Copyright Law is authorized by SPIE subject to payment of copying fees. The Transactional Reporting Service base fee for this volume is $\$ 18.00$ per article (or portion thereof), which should be paid directly to the Copyright Clearance Center (CCC), 222 Rosewood Drive, Danvers, MA 01923. Payment may also be made electronically through CCC Online at copyright.com. Other copying for republication, resale, advertising or promotion, or any form of systematic or multiple reproduction of any material in this book is prohibited except with permission in writing from the publisher. The CCC fee code is 0277-786X/11/ \$18.00.

Printed in the United States of America.

Publication of record for individual papers is online in the SPIE Digital Library.

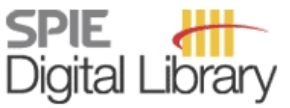

SPIEDigitalLibrary.org

Paper Numbering: Proceedings of SPIE follow an e-First publication model, with papers published first online and then in print and on CD-ROM. Papers are published as they are submitted and meet publication criteria. A unique, consistent, permanent citation identifier (CID) number is assigned to each article at the time of the first publication. Utilization of CIDs allows articles to be fully citable as soon they are published online, and connects the same identifier to all online, print, and electronic versions of the publication. SPIE uses a six-digit CID article numbering system in which:

- The first four digits correspond to the SPIE volume number.

- The last two digits indicate publication order within the volume using a Base 36 numbering system employing both numerals and letters. These two-number sets start with 00, 01, 02, 03, 04, $05,06,07,08,09,0 A, 0 B \ldots 0 Z$, followed by 10-1Z, 20-2Z, etc.

The CID number appears on each page of the manuscript. The complete citation is used on the first page, and an abbreviated version on subsequent pages. Numbers in the index correspond to the last two digits of the six-digit CID number. 


\section{Contents}

xi Conference Committee

$\mathrm{xV} \quad$ Single-crystal YAG fiber optics for the transmission of high energy laser energy (Presented in Conference 7912) [789415]

X. S. Zhu, Fudan Univ. (China) and Rutgers Univ. (United States); J. A. Harrington,

B. T. Laustsen, Rutgers Univ. (United States); L. G. DeShazer, Lightwave Energenics LLC (United States)

xxiii Microfabrication by optical tweezers (Plenary Paper) [792102]

R. Ghadiri, T. Weigel, C. Esen, A. Ostendorf, Ruhr-Univ. Bochum (Germany)

\section{SESSION 1 SPACE-QUALIFIED LASERS I}

791203 Wavelength and time-multiplexed multi-channel lidar transmitter for topographic mapping mission [7912-02]

Y. Chen, F. Kimpel, J.-L. Fouron, Fibertek, Inc. (United States); J. Chen, NASA Goddard Space Flight Ctr. (United States); S. Gupta, Fibertek, Inc. (United States)

\section{SESSION 2 SPACE-QUALIFIED LASERS II}

791205 Non-linear optical frequency conversion crystals for space applications (Invited Paper) [7912-04]

A. Ciapponi, W. Riede, Deutsches Zentrum für Luft- und Raumfahrt e.V. (Germany);

G. Tzeremes, ESA / ESTEC (Netherlands); H. Schröder, P. Mahnke, Deutsches Zentrum für Luftund Raumfahrt e.V. (Germany)

791206 Development of a low SWaP laser transmitter for atmospheric lidar applications [7912-05] N. S. Prasad, NASA Langley Research Ctr. (United States); A. Rosiewicz, S. M. Coleman, EM4, Inc. (United States)

\section{SESSION 3 THIN DISK LASERS I}

791209 From multi kW continuous wave to multi MW femtosecond pulses: recent developments exploiting disk laser technology [7912-08]

S. Weiler, M. Holzer, TRUMPF Laser- und Systemtechnik GmbH (Germany);

P. Graham, J. Stollhof, TRUMPF Inc. (United States); D. Havrilla, TRUMPF Inc. Laser Technology Ctr. (United States)

$79120 \mathrm{~A} \quad 50 \mathrm{~W}$ thin-disk laser with variable pulse duration [7912-09]

M. Larionov, F. Dausinger, Dausinger + Giesen GmbH (Germany) 
7912 OB Thermal and stress characterization of various thin disk laser configurations at room temperature [7912-11]

N. Vretenar, The Univ. of New Mexico (United States); T. Carson, Air Force Research Lab. (United States); P. Peterson, T. Lucas, Boeing LTS Inc. (United States); T. C. Newell, W. P. Latham, Air Force Research Lab. (United States)

\section{SESSION 4 THIN DISK LASERS II}

7912 OC High power thin disk Ho:YAG laser [7912-12]

J. Speiser, G. Renz, A. Giesen, German Aerospace Ctr. (Germany)

7912 OD Optical extraction model and optimal outcoupling for a CW quasi-three level thin disk laser [7912-14]

D. A. Copeland, Aqwest, LLC (United States)

7912 OE Fast wavelength switching of a single-frequency disk laser amplifier [7912-15]

W. Paa, V. Wagner, T. Zeuner, M. Franke, IPHT Jena (Germany)

7912 OF High power disk lasers: advances and applications [7912-16]

D. Havrilla, TRUMPF Inc. (United States); M. Holzer, TRUMPF Laser- und Systemtechnik GmbH (Germany)

\section{SESSION $5 \quad$ VISIBLE LASERS I: JOINT SESSION WITH CONFERENCE 7917}

$79120 \mathrm{G}$ A compact optically pumped semiconductor laser emitting at $593 \mathrm{~nm}$ [7912-17]

R. von Elm, Coherent Lubeck GmbH (United States); S. Offen, Laserlabor (Germany); W. Seelert, V. Ostroumov, D. Mohrenstecher, Coherent Lubeck GmbH (Germany); J. Brunn, Laserlabor (Germany)

SESSION 6 VISIBLE LASERS II: JOINT SESSION WITH CONFERENCE 7917

$7912 \mathrm{OH}$ Harmonic generation with fiber MOPAs and solid state lasers: technical challenges, state-of-the-art comparison, and future developments [7912-18] A. Starodoumov, N. Hodgson, Coherent, Inc. (United States)

$791201 \quad$ Low noise Raman lasers for yellow-orange spectrum coverage [7912-19] N. Landru, J. Rouvillain, G. Le Bail, T. Georges, Oxxius SA (France)

7912 0J $575 \mathbf{n m}$ laser oscillation in $\mathrm{Dy}^{3+}$-doped waterproof fluoro-aluminate glass fiber pumped by violet GaN laser diodes [7912-20]

Y. Fujimoto, Osaka Univ. (Japan); O. Ishii, M. Yamazaki, Sumita Optical Glass, Inc. (Japan)

7912 OK Efficient frequency conversion of pulsed microchip and fiber laser radiation in PPSLT [7912-21]

B. Jungbluth, S. Nyga, E. Pawlowski, T. Fink, J. Wüppen, Fraunhofer-Institut für Lasertechnik (Germany) 
7912 OL Yb-doped ultrafast thin disk lasers (Invited Paper) [7912-22]

T. Südmeyer, C. R. E. Baer, ETH Zurich (Switzerland); C. Kränkel, ETH Zurich (Switzerland) and Univ. Hamburg (Germany); C. J. Saraceno, O. H. Heckl, M. Golling, ETH Zurich (Switzerland); R. Peters, K. Petermann, G. Huber, Univ. Hamburg (Germany); U. Keller, ETH Zurich (Switzerland)

7912 OM Highly flexible ultrafast laser system with 260W average power [7912-23]

T. Mans, J. Dolkemeyer, AMPHOS GmbH (Germany); P. Russbüldt, Fraunhofer ILT (Germany); C. Schnitzler, AMPHOS GmbH (Germany)

7912 ON Picosecond laser with 400W average power and 1mJ pulse energy [7912-24]

D. Li, K. Du, EdgeWave GmbH (Germany)

SESSION $8 \quad$ ULTRAFAST LASERS II

$79120 Q \quad$ Yb-doped ultrafast solid state lasers (Invited Paper) [7912-27]

D. N. Papadopoulos, LCFIO, UMR CNRS, Univ. Paris-SUd (France) and ILE, UMS CNRS, Ecole Polytechnique, ENSTA ParisTech, Institut d'Optique, Univ. Paris-Sud (France); P. Georges, LCFIO, UMR CNRS, Univ. Paris-Sud (France); P. Camy, J.-L. Doualan, R. Moncorgé, CIMAP, Univ. de Caen (France); B. Viana, P. Goldner, LCMCP (France); F. Druon, LCFIO, UMR CNRS, Univ. Paris-Sud (France)

7912 OR 1100 W Yb:YAG femtosecond Innoslab amplifier (Invited Paper) [7912-28]

P. Russbueldt, Fraunhofer-Institut für Lasertechnik (Germany); T. Mans, RWTH Aachen Univ. (Germany); H.-D. Hoffmann, Fraunhofer-Institut für Lasertechnik (Germany); R. Poprawe, Fraunhofer-Institut für Lasertechnik (Germany) and RWTH Aachen Univ. (Germany)

7912 OS Diode-pumped $\mathrm{Yb}: \mathrm{CaF}_{2}$ regenerative amplifier [7912-29]

S. Ricaud, LCFIO, CNRS, Univ. Paris-Sud (France) and Amplitude Systèmes (France); F. Druon, LCFIO, CNRS, Univ. Paris-Sud (France); D. N. Papadopoulos, LCFIO, CNRS, Univ. Paris-Sud (France) and ILE, CNRS, Univ. Paris-Sud (France); P. Camy, J. Doualan, R. Moncorgé, CIMAP, CNRS-ENSICAEN, Univ. de Caen (France); M. Delaigue, Y. Zaouter, A. Courjaud, Amplitude Systèmes (France); P. Georges, LCFIO, CNRS, Univ. Paris-Sud (France); E. Mottay, Amplitude Systèmes (France)

7912 OT Steady state mode-locking of the $\mathrm{Nd} \mathrm{YVO}_{4}$ laser operating on the $1.34 \mu \mathrm{m}$ transition using intracavity SHG in BIBO or PPMgSLT [7912-30]

H. Iliev, I. Buchvarov, V. Alexandrov, Sofia Univ. (Bulgaria); S. Kurimura, National Institute for Materials Science (Japan); V. Petrov, Max-Born-Institute for Nonlinear Optics and Ultrafast Spectroscopy (Germany)

\section{SESSION 9 Q-SWITCHED LASERS}

7912 OU A Joule-class, TEMoo spatial profile, narrow-linewidth laser system [7912-31]

A. Vaupel, N. Bodnar, M. Hemmer, M. Richardson, Univ. of Central Florida (United States)

7912 OV UV power scaling of INNOSLAB lasers [7912-32]

D. Li, S. Fu, J. Chen, K. Du, EdgeWave GmbH (Germany) 
7912 OW Passive synchronized Q-switching between a quasi-three-level and a four-level laser [7912-33]

H. P. H. Cheng, P. Tidemand-Lichtenberg, O. B. Jensen, P. E. Andersen, P. M. Petersen,

C. Pedersen, Technical Univ. of Denmark (Denmark)

7912 0X Optically triggered Cr:YAG Q-switched Nd:YAG laser [7912-34]

B. Cole, A. Hays, J. Lei, B. Schilling, L. Goldberg, U.S. Army, RDECOM CERDEC (United States)

$79120 Z$ High power $808 \mathrm{~nm}$ VCSEL arrays for pumping of compact pulsed high energy Nd:YAG lasers operating at $946 \mathrm{~nm}$ and $1064 \mathrm{~nm}$ for blue and UV light generation [7912-36] R. van Leeuwen, Y. Xiong, L. S. Watkins, J.-F. Seurin, G. XU, Q. Wang, C. Ghosh, Princeton Optronics, Inc. (United States)

791210 High-average power $\mathrm{Nd}^{\mathrm{Y}} \mathrm{YVO}_{4}$ regenerative amplifier seeded by a gain switched diode laser [7912-37]

M. Lührmann, F. Harth, C. Theobald, T. Ulm, Photonik-Zentrum Kaiserslautern e.V. (Germany); R. Knappe, A. Nebel, LUMERA LASER GmbH (Germany); A. Klehr, G. Erbert,

Ferdinand-Braun-Institut (Germany); J. A. L'huillier, Photonik-Zentrum Kaiserslautern e.V. (Germany)

\section{SESSION 11 OPTICS, FIBERS, AND METROLOGY}

791213 Developing a more useful surface quality metric for laser optics [7912-40]

Q. Turchette, T. Turner, Research Electro-Optics, Inc. (United States)

791214 Novel concept for large distance ultrashort pulse fiber delivery without pre-chirping [7912-41]

T. Le, G. Tempea, FEMTOLASERS Produktions GmbH (Austria); K. G. Jespersen, OFS Fitel Denmark (Denmark); H. Pühringer, K. Wiesauer, RECENDT GmbH (Austria)

791215 Beam steering mirror for fine adjustment of visible laser single mode fiber coupling based on a birefringent crystal [7912-42]

C. Kannengiesser, W. Seelert, R. v. Elm, S. Kubasiak, Coherent Lubeck GmbH (Germany); R. Austin, Coherent, inc. (United States)

$791216 \quad$ All-in-quartz optics for low focal shifts [7912-43]

M. Blomqvist, O. Blomster, M. Pålsson, S. Campbell, Optoskand AB (Sweden); F. Becker, W. Rath, Rofin-Sinar Laser GmbH (Germany)

791217 Novel all-hardware closed-loop adaptive optics system using massively-parallel neural processing [7912-44]

M. Eichhorn, A. Pichler, P. Raymond, French-German Research Institute of Saint-Louis (France) 
7912 1B LD pumped high efficiency, high power Tm:YLF laser with adhesive-free bond laser composites [7912-48]

X. Mu, H. Meissner, H.-C. Lee, Onyx Optics Inc. (United States)

7912 1C Energy scaling of room temperature Fe ${ }^{2+}: Z n S e$ gain-switched $4.3 \mu \mathrm{m}$ laser [7912-49] N. Myoung, D. V. Martyshkin, V. V. Fedorov, A. Martinez, S. B. Mirov, The Univ. of Alabama at Birmingham (United States)

7912 1D Miniaturized high power Er:YAG solid state laser pumped by a single laser diode bar [7912-50]

M. Krejci, Oclaro, Inc. (Switzerland); A. Heinrich, Pantec Biosolutions AG (Liechtenstein); J. Müller, Oclaro, Inc. (Switzerland); T. Bragagna, Pantec Biosolutions AG (Liechtenstein); N. Lichtenstein, Oclaro, Inc. (Switzerland)

$79121 \mathrm{E} \quad$ Energy scaling of nanosecond gain-switched $\mathrm{Cr}^{2+}: Z n S e$ lasers [7912-51]

V. V. Fedorov, The Univ. of Alabama at Birmingham (United States) and IPG Photonics Corp. (United States); I. S. Moskalev, M. S. Mirov, IPG Photonics Corp. (United States); S. B. Mirov, The Univ. of Alabama at Birmingham (United States) and IPG Photonics Corp. (United States); T. J. Wagner, M. J. Bohn, Air Force Institute of Technology (United States); P. A. Berry, K. L. Schepler, Air Force Research Lab. (United States)

\section{SESSION 13 MID-INFRARED LASERS II}

$79121 \mathrm{~F}$ The effect of the nature of the rare earth additive on chalcogenide glass stability [7912-52] Z. Tang, D. Furniss, S. Sujecki, T. M. Benson, A. B. Seddon, The Univ. of Nottingham (United Kingdom)

$79121 \mathrm{G} \quad$ High-power diode-pumped Q-switched $\mathrm{Er}^{3+}:$ YAG single-crystal fiber laser [7912-53] I. Martial, Lab. Charles Fabry, CNRS, Univ. Paris-Sud (France) and FiberCryst SAS (France); J. Didierjean, N. Aubry, FiberCryst SAS (France); F. Balembois, P. Georges, Lab. Charles Fabry, CNRS, Univ. Paris-Sud (France)

$79121 \mathrm{H} \quad$ Cr:ZnSe planar waveguide mid-IR laser [7912-54]

J. E. Willimas, The Univ. of Alabama at Birmingham (United States); D. V. Martyshkin, V. V. Fedorov, The Univ. of Alabama at Birmingham (United States) and Photonics Innovations, Inc. (United States); I. S. Moskalev, Photonics Innovations, Inc. (United States); R. P. Camata, The Univ. of Alabama at Birmingham (United States); S. B. Mirov, The Univ. of Alabama at Birmingham (United States) and Photonics Innovations, Inc. (United States)

$791211 \quad$ Mid-IR lasing of $\mathrm{Cr}: Z n S e / \mathrm{As}_{2} \mathrm{~S}_{3}: \mathrm{As}_{2} \mathrm{Se}_{3}$ composite materials [7912-55]

D. V. Martyshkin, V. V. Fedorov, The Univ. of Alabama at Birmingham (United States) and IPG Photonics Corp. (United States); J. T. Goldstein, Air Force Research Lab. (United States); S. B. Mirov, The Univ. of Alabama at Birmingham (United States) and IPG Photonics Mid-Infrared Lasers (United States) 
$79121 \mathrm{~K}$ Improved characterization of transmitted wavefront error on CADB epoxy-free bonded solid state laser materials [7912-57]

N. Traggis, N. Claussen, Precision Photonics Corp. (United States); A. Bayramian, K. Schaffers, B. Deri, Lawrence Livermore National Lab. (United States)

$79121 \mathrm{~L} \quad$ Lasing properties of new $\mathrm{Yb}$-doped borate compounds with varying gadolinium and yttrium concentration [7912-58]

I. Manek-Hönninger, Univ. Bordeaux 1 (France); M. Chavoutier, V. Jubera, ICMCB-CNRS, Univ. Bordeaux 1 (France); D. Descamps, Univ. Bordeaux 1 (France); P. Veber, M. Velazquez, A. Garcia, ICMCB-CNRS, Univ. Bordeaux 1 (France); L. Canioni, Univ. Bordeaux 1 (France)

$79121 \mathrm{~N}$ Compositionally tuned $\mathrm{Nd}$ : $\left(\mathrm{Y}_{\mathrm{x}} \mathrm{LU}_{1-\mathrm{x}}\right)_{3} \mathrm{Ga}_{5} \mathrm{O}_{12}$-laser at $935 \mathrm{~nm}$ for $\mathrm{H}_{2} \mathrm{O}$-dial systems [7912-60] J. Löhring, M. Schlößer, H.-D. Hoffmann, Fraunhofer-Institut für Lasertechnik (Germany)

791210 Diode-pumped, cryogenically cooled $\mathrm{Yb}: \mathrm{CaF}_{2}$ for high efficient and high power laser [7912-61]

S. Ricaud, LCFIO, CNRS, Univ. Paris-Sud (France) and Amplitude Systèmes (France);

D. N. Papadopoulos, A. Pellegrina, ILE, CNRS, Univ. Paris-Sud (France); P. Camy,

J. L. Doualan, R. Moncorgé, CIMAP, CNRS-ENSICAEN, Univ. de Caen (France); A. Courjaud, E. Mottay, Amplitude Systèmes (France); P. Georges, F. Druon, LCFIO, CNRS, Univ. Paris-Sud (France)

7912 IP High power Raman diamond laser [7912-62]

J.-P. M. Feve, M. J. Bohn, J. K. Brasseur, K. Shortoff, Directed Energy Solutions (United States)

\section{SESSION 15 LASER MATERIALS II}

$79121 Q \quad$ Ceramic laser materials (Invited Paper) [7912-63]

J. Sanghera, B. Shaw, W. Kim, G. Villalobos, C. Baker, J. Frantz, U.S. Naval Research Lab. (United States); M. Hunt, Univ. Research Foundation (United States); B. Sadowski, Global Defense Technology \& Systems, Inc. (United States); I. Aggarwal, U.S. Naval Research Lab. (United States)

$79121 \mathrm{R} \quad$ Last advances in $\mathrm{Yb}^{3+}$ doped $\mathrm{CaF}_{2}$ ceramics synthesis (Invited Paper) [7912-64] M. Mortier, A. Lyberis, A. Saganuma, A. Stevenson, D. Vivien, P. Gredin, Lab. de Chimie de la Matière Condensée de Paris, CNRS, Univ. Pierre et Marie Curie (France); G. Patriarche, Lab. de Photonique et de Nanostructure, CNRS (France)

$79121 \mathrm{~T}$ Processing and transparency of polycrystalline yttrium aluminum garnet (YAG) fiber for optical applications [7912-66]

H. J. Kim, UES, Inc. (United States); G. Fair, Air Force Research Lab. (United States); H. Lee, K. Keller, T. A. Parthasarathy, UES, Inc. (United States); R. Hay, Air Force Research Lab. (United States) 
$79121 \mathrm{~W}$ Precision Fresnel attenuator of the beam power of laser radiation [7912-68] J. Owsik, Military Univ. of Technology (Poland); A. A. Kovalev, S. A. Moskalyuk, E. B. Yankevich, All-Russian Research Institute for Optical and Physical Measurement (Russian Federation); A. Rembielińska, LOT Polish Airlines (Poland)

7912 1X 747 nm Pr:YAP microchip-laser output characteristics [7912-69] M. Fibrich, H. Jelínková, J. Šulc, Czech Technical Univ. in Prague (Czech Republic); K. Nejezchleb, V. Škoda, Crytur Ltd. (Czech Republic)

$79121 Y \quad$ Design of micro-second pulsed laser mode for ophthalmological CW self-raman laser [7912-70]

A. D. Mota, Opto Eletrônica S.A. (Brazil) and Univ. de São Paulo (Brazil); G. Rossi, T. A. Ortega, G. Z. Costal, Y. C. Fontes, F. M. M. Yasuoka, M. A. Stefani, J. C. de Castro N., Opto Eletrônica S.A. (Brazil); M. S. V. Paiva, Univ. de São Paulo (Brazil)

$79121 Z$ The research of the laser facula of laser ranger finder in the far distance [7912-71] R. Fu, C. Su, M. Li, Y. Guo, Y. Cheng, B. Chang, Nanjing Univ. of Science \& Technology (China)

791220 Compact intracavity frequency doubled diode laser at 465 nm [7912-72]

K. Li, N. J. Copner, Univ. of Glamorgan (United Kingdom); C. B. E. Gawith, Covesion Ltd. (United Kingdom); I. G. Knight, Oclaro Technology Ltd. (United Kingdom); H.-U. Pfeiffer, Oclaro AG (Switzerland); B. Musk, Gooch \& Housego (United Kingdom)

791221 Passively Q-switched quasi-continuously pumped 2.4\% Nd:YAG laser in a bounce geometry [7912-73]

M. Jelínek, V. Kubeček, M. Čech, P. Hiršl, Czech Technical Univ. in Prague (Czech Republic)

791222 Influence of V:YAG saturable absorber orientation on linearly polarized laser Q-switching [7912-74]

J. Šulc, T. Koutný, H. Jelínková, Czech Technical Univ. in Prague (Czech Republic);

K. Nejezchleb, V. Škoda, Crytur Ltd. (Czech Republic)

$791223 \quad$ Noise performances of a high-power picosecond Nd:YVO4 Oscillator [7912-75] M.-C. Nadeau, Univ. Bordeaux 1 (France) and Thales Optronique SA (France); S. Petit, P. Balcou, S. Montant, Univ. Bordeaux 1 (France); C. Simon-Boisson, Thales Optronique SA (France)

791224 Role of UV illumination for enabling cold temperature operation of a $\mathrm{LiNbO}_{3} \mathrm{Q}$-switched Nd:YAG laser [7912-76]

B. Cole, V. King, J. Leach, L. Goldberg, U.S. Army RDECOM CERDEC (United States)

791225 LD-pumped soliton and non-soliton mode-locked operations of $\mathrm{Yb}:\left(\mathrm{Gd}_{1-\mathrm{x}} \mathrm{Yx}\right)_{2} \mathrm{SiO}_{5}(\mathrm{x}=\mathbf{0 . 5 )}$ laser [7912-78]

J. He, X. Liang, J. Li, L. Zheng, L. Su, J. Xu, Z. Xu, Shanghai Institute of Optics and Fine Mechanics (China) 
791226 High power red laser oscillation in $\mathrm{Pr}^{3+}$-doped waterproof fluoro-aluminate glass fiber excited by a GaN laser diode [7912-79]

J. Nakanishi, T. Yamada, NIDEK Co., Ltd. (Japan); Y. Fujimoto, Osaka Univ. (Japan); O. Ishii, M. Yamazaki, Sumita Optical Glass, Inc. (Japan)

791228 Low threshold, high power passively mode-locked Yb:SSO laser [7912-81]

J. Li, X. Liang, J. He, Shanghai Institute of Optics and Fine Mechanics (China); L. Zheng, L. Su, J. XU, Shanghai Institute of Ceramics (China)

791229 Effects of $\mathbf{Y}$-irradiation on optical, electrical, and laser characteristics of pure and transition metal doped II-VI semiconductors [7912-83]

T. Konak, M. Tekavec, V. V. Fedorov, S. B. Mirov, The Univ. of Alabama at Birmingham (United States)

7912 2A A single frequency Nd:YAG Q-switched laser with timely controllable firing time by a gated Pockels cell [7912-84]

F. F. Wu, A. Khizhnyak, V. Markov, MetroLaser, Inc. (United States)

7912 2B A hybrid fiber/solid state regenerative amplifier with tunable pulse widths for satellite laser ranging [7912-85]

D. Poulios, American Univ. (United States); D. B. Coyle, NASA Goddard Space Flight Ctr. (United States); R. B. Kay, American Univ. (United States); P. R. Stysley, NASA Goddard Space Flight Ctr. (United States)

7912 2D Fe:ZnSe laser: comparison of active materials grown by two different methods [7912-87] M. E. Doroshenko, A. M. Prokhorov General Physics Institute (Russian Federation);

H. Jelínková, Czech Technical Univ. in Prague (Czech Republic); T. T. Basiev, A. M. Prokhorov General Physics Institute (Russian Federation); M. Jelínek, P. Koranda, M. Němec, Czech Technical Univ. in Prague (Czech Republic); V. K. Komar, A. S. Gerasimenko, Institute for Single Crystals (Ukraine); V. V. Badikov, D. V. Badikov, Kuban State Technological Univ. (Russian Federation); D. Vyhlídal, J. Stoklasa, Czech Technical Univ. in Prague (Czech Republic)

$79122 \mathrm{~F} \quad$ Efficient compensation of thermal birefringence of a flash-lamp pumped Nd:YAG laser by a simple but novel method [7912-89]

S. Mondal, S. Datta, S. Dey, S. Bera, S. P. Singh, P. K. Datta, Indian Institute of Technology, Kharagpur (India)

7912 2G Intense red upconversion fluorescence emission in NIR-excited erbium-ytterbium doped laponite-derived phosphor [7912-90]

A. F. da Silva, D. S. Moura, A. S. Gouveia-Neto, E. A. Silva, Jr., L. A. Bueno, E. B. Costa, Univ. Federal Rural de Pernambuco (Brazil); E. N. Azevedo, Ministério da Ciência e Tecnologia (Brazil)

$79122 \mathrm{H} \quad$ Properties of rare earth doped thin film dielectric layers for upconversion laser waveguides [7912-91]

S. J. Pearce, M. D. B. Charlton, G. J. Parker, J. S. Wilkinson, Univ. of Southampton (United Kingdom)

Author Index 


\title{
Conference Committee
}

\author{
Symposium Chairs
}

Friedhelm Dorsch, TRUMPF GmbH \& Company KG (Germany)

Alberto Piqué, U.S. Naval Research Laboratory (United States)

Symposium Cochairs

Donald J. Harter, IMRA America, Inc. (United States)

Peter R. Herman, University of Toronto (Canada)

Program Track Chair

Gregory J. Quarles, BE Meyers \& Company Inc. (United States)

Conference Chairs

W. Andrew Clarkson, University of Southampton (United Kingdom)

Norman Hodgson, Coherent, Inc. (United States)

Ramesh Shori, Naval Air Warfare Center Weapons Division (United States)

Program Committee

Santanu Basu, Sparkle Optics Corporation (United States)

Marc Eichhorn, Institut Franco-Allemand de Recherches de Saint-Louis (France)

Adolf Giesen, Deutsches Zentrum für Luft- und Raumfahrt e.V. (Germany)

Hans-Dieter Hoffmann, Fraunhofer-Institut für Lasertechnik (Germany)

Helena Jelinková, Czech Technical University in Prague (Czech Republic)

Ursula Keller, ETH Zurich (Switzerland)

Jacob I. Mackenzie, University of Southampton (United Kingdom)

Michio Oka, Sony Corporation (Japan)

Narasimha S. Prasad, NASA Langley Research Center (United States)

Martin C. Richardson, CREOL, The College of Optics and Photonics,

University of Central Florida (United States)

Wolf Seelert, Coherent Lubeck GmbH (Germany)

David E. Spence, Spectra-Physics $\AA^{\circledR}$, a Newport Corporation Brand (United States)

David H. Titterton, Defence Science and Technology Laboratory (United Kingdom) 
Session Chairs

1 Space-qualified Lasers I

Ramesh K. Shori, Naval Air Warfare Center Weapons Division (United States)

2 Space-qualified Lasers II

Ramesh K. Shori, Naval Air Warfare Center Weapons Division (United States)

3 Thin Disk Lasers I

Hans-Dieter Hoffmann, Fraunhofer-Institut für Lasertechnik (Germany)

4 Thin Disk Lasers II

Santanu Basu, Sparkle Optics Corporation (United States)

$5 \quad$ Visible Lasers I: Joint Session with Conference 7917

W. Andrew Clarkson, University of Southampton (United Kingdom)

Andrei V. Shchegrov, Spectralus Corporation (United States)

$6 \quad$ Visible Lasers II: Joint Session with Conference 7917

Andrei V. Shchegrov, Spectralus Corporation (United States)

Wolf Seelert, Coherent Lübeck GmbH (Germany)

$7 \quad$ Ultrafast Lasers I

W. Andrew Clarkson, University of Southampton (United Kingdom)

8 Ultrafast Lasers II

David E. Spence, Spectra-Physics ${ }^{\circledR}$, a Newport Corporation Brand (United States)

9 Q-switched Lasers

Helena Jelinková, Czech Technical University in Prague (Czech Republic)

10 Pulsed Amplified Sources: Joint Session with Conference 7914

Norman Hodgson, Coherent, Inc. (United States)

Dahv A. V. Kliner, JDSU (United States)

11 Optics, Fibers, and Metrology

David H. Titterton, Defence Science and Technology Laboratory (United Kingdom)

12 Mid-Infrared Lasers I

Ramesh K. Shori, Naval Air Warfare Center Weapons Division (United States) 
13 Mid-Infrared Lasers II

Helena Jelinková, Czech Technical University in Prague (Czech

Republic)

14 Laser Materials I

Marc Eichhorn, Institut Franco-Allemand de Recherches de Saint-Lovis (France)

15 Laser Materials II

Jacob I. Mackenzie, University of Southampton (United Kingdom) 
Downloaded From: https://www.spiedigitallibrary.org/conference-proceedings-of-spie on 26 Apr 2023

Terms of Use: https://www.spiedigitallibrary.org/terms-of-use 